\title{
Avaliação da sexualidade de pacientes com câncer do colo do útero em Sergipe
}

\author{
Evaluation of the sexuality of cervical cancer patients in Sergipe \\ Evaluación de la sexualidad de pacientes con cáncer de cuello uterino en Sergipe
}

Recebido: 26/01/2021 | Revisado: 01/02/2021 |Aceito: 15/02/2021 | Publicado: 21/02/2021

Yasmin Melo Toledo

ORCID: https://orcid.org/0000-0003-0468-0544 Universidade Tiradentes, Brasil

E-mail: yasmin.se@ hotmail.com

Marina de Pádua Nogueira Menezes

ORCID: https://orcid.org/0000-0002-3936-7470

Universidade Tiradentes, Brasil

E-mail: marinapnogueira@yahoo.com.br

Erica Silva Barros

ORCID: https://orcid.org/0000-0003-1073-7738

Universidade Federal de Sergipe, Brasil

E-mail: ericabarrosmed@bol.com.br

\begin{abstract}
Resumo
Objetivo: Analisar os impactos do câncer de colo de útero na sexualidade das mulheres, ressaltando a importância desta para a qualidade de vida. Métodos: Trata-se de um estudo epidemiológico, descritivo e analítico, com coleta de dados de caráter transversal. Foram realizadas 80 entrevistas no ambulatório de oncologia do Hospital de Urgência de Sergipe em mulheres com câncer de colo de útero em tratamento ou já tratadas, utilizando-se dois questionários. O primeiro abordou aspectos socioeconômicos e demográficos. O segundo, o Quociente Sexual versão Feminina (QS-F). Os dados foram analisados por estatística descritiva com auxílio do software Epi-Info versão 7.0. Por se tratar de pesquisa com seres humanos, o trabalho foi submetido e aprovado pelo Comitê de Ética em Pesquisa. Resultados: A média de idade foi 46,8 anos $( \pm 13,2)$. A escolaridade apresentou-se com prevalência de indivíduos com nível médio completo 25\% ( $\mathrm{n}=20)$. Quanto ao estado civil, nível sócio econômico e profissão, 36,3\% ( $\mathrm{n}=29)$ declararam-se casadas, $51,2 \%(\mathrm{n}=41)$ do estrato social de classe média e 33, $8(\mathrm{n}=27)$ exerciam atividade de "do lar". O QS-F apresentou média geral de 45,5 pontos $( \pm 23,9)$, variando de 10 a 84 pontos. Adotada a classificação estratificada, observou-se que boa parte das mulheres, $85 \%(\mathrm{n}=68)$, foram classificadas como regular ou inferior. Foi evidenciado diferença estatisticamente significante entre os estratos do QS-F e o nível socioeconômico. Conclusão: $\mathrm{O}$ presente trabalho corrobora com a literatura utilizada em vários âmbitos, uma vez que a maioria das mulheres questionadas mostrou função sexual baixa e insatisfatória de acordo com o QS-F.
\end{abstract}

Palavras-chave: Sexualidade; Oncologia; Neoplasias do colo do útero.

\begin{abstract}
Objective: Analyze the impacts of cervical cancer on women's sexuality, evidencing its importance for quality of life. Methods: This is an epidemiological, descriptive and analytical study, with cross-sectional data collection. Eighty women with cervical câncer, undergoing treatment or already treated, were interviwed in the oncology clinic of Hospital de Urgência de Sergipe, through two questionnaires. The first one results in socioeconomic and demographic aspects. The second one, in Female Sexual Quotient version (QS-F). The data was analyzed using descriptive statistics using the Epi-Info version 7.0 software. The work was submitted and approved by the Research Ethics Committee considering it is a rsearch oh human beigns. Results: The average age was 46.8 years $( \pm 13.2)$. Education level was $25 \%$ higher $(\mathrm{n}=20)$. Regarding marital status, socioeconomic status and professional one, $36.3 \%(\mathrm{n}=29)$ declared themselves married, $51.2 \%(\mathrm{n}=41)$ from the middle class and $33.8(\mathrm{n}=27)$ doing "home" activity. The QS-F had an overall average of 45.5 points $( \pm 23.9)$, ranging from 10 to 84 points. Adopting the stratified classification, it was observed that a big number of women, $85 \%(\mathrm{n}=68)$, were classified as regular or inferior. There was a statistically significant difference between the strata of the QS-F and the socioeconomic level. Conclusion: The present study corroborates with the literature used in several areas, since most of the women questioned showed low and unsatisfactory sexual function according to the QS-F.
\end{abstract}

Keywords: Sexuality; Medical oncology; Uterine cervical neoplasms. 


\section{Resumen}

Objetivo: Analizar los impactos del cáncer de cuello uterino en la sexualidad de las mujeres, destacando su importancia para la calidad de vida. Métodos: Se trata de un estudio epidemiológico, descriptivo y analítico, con recolección de datos transversal. Se realizaron ochenta entrevistas en el ambulatorio de oncología del Hospital de Urgência de Sergipe en mujeres con cáncer de cuello uterino en tratamiento o ya tratadas, mediante dos cuestionarios. El primero abordó aspectos socioeconómicos y demográficos. El segundo, la versión del Cociente Sexual Femenino (QS-F). Los datos se analizaron mediante estadística descriptiva utilizando el software Epi-Info versión 7.0. Al tratarse de una investigación con seres humanos, el trabajo fue presentado y aprobado por el Comité de Ética en Investigación. Resultados: La edad media fue de 46,8 años $( \pm 13,2)$. El nivel educativo fue un $25 \%$ superior $(n=20)$. En cuanto al estado civil, nivel socioeconómico y profesión, el 36,3\% (n = 29) se declaró casado, el 51,2\% $(\mathrm{n}=41)$ de clase media y el 33,8 $(\mathrm{n}=27)$ actividad "doméstica". El QS-F tuvo un promedio general de 45,5 puntos $( \pm 23,9)$, con un rango de 10 a 84 puntos. Adoptando la clasificación estratificada, se observó que un buen número de mujeres, el $85 \%(n=68)$, fueron clasificadas como regulares o inferiores. Hubo una diferencia estadísticamente significativa entre los estratos del QS-F y el nivel socioeconómico. Conclusión: El presente estudio corrobora la literatura utilizada en varias áreas, ya que la mayoría de las mujeres encuestadas mostró una función sexual baja e insatisfactoria según la QS-F.

Palabras clave: Sexualidad; Oncología médica; Neoplasias del cuello uterino.

\section{Introdução}

As discussões sobre sexualidade sempre envolvem aspectos e valores que a tornam um campo de difícil arguição e repleto de paradigmas ideológicos e conceituais. Quando há um fator agravante para esta discussão, o tema torna-se ainda mais complexo e impeditivo, como é o caso da sexualidade em pacientes com câncer do colo de útero. Assim, desvelar problemáticas deste campo de estudo é de fundamental importância para o adequado manejo da informação e direcionamento terapêutico destes pacientes.

O câncer do colo uterino é um dos mais relevantes a nível mundial, com incidência estimada maior que meio milhão de casos novos por ano. Sabe-se ainda que foi responsável por aproximadamente meio milhão de casos novos, além de 311 mil mortes em todo o mundo em 2017. Dessas mortes, a Organização das Nações Unidas (OMS) afirma que cerca de 85\% aconteceram em países de média e baixa renda. (World Health Organization, 2017)

De acordo com as estimativas de incidência de câncer em 2018, esperou-se para o Brasil mais de 16.000 novos casos de câncer do colo uterino, com um risco estimado de 15,38 casos para cada 100 mil mulheres. Sem considerar os tumores de pele não melanoma, o câncer do colo do útero foi o mais incidente na região Norte (26,24/ 100 mil). Nas regiões Centro-Oeste (12,35/ 100 mil) e Nordeste (16,10/ 100 mil), foi o segundo mais frequente. Na região Sul (12,60/100 mil), o quarto e, na região Sudeste (08,61 /100 mil), o quinto mais frequente (INCA, 2018a).

O tipo histológico mais comum do câncer do colo do útero é o carcinoma de células escamosas, representando cerca de $85 \%$ a $90 \%$ de todos os casos, seguido pelo adenocarcinoma. O principal fator de risco para o desenvolvimento do câncer do colo do útero é a infecção pelo Papilomavírus Humano (HPV). Contudo, essa infecção, por si só, não representa uma causa suficiente para o surgimento da neoplasia, fazendo-se necessária a persistência deste vírus no organismo, atuando juntamente com outros fatores de risco como idade, aspectos sexuais, paridade, tabagismo (De Mendonça et al., 2019; Saslow et al., 2012)

Antigamente, acreditava-se que o Papiloma Vírus Humano (HPV) era apenas um fator de risco para o desenvolvimento de Neoplasia Maligna Cervical. Entretanto, atualmente sabe-se que, na verdade, o HPV representa a principal etiologia dessa doença, constituindo um fator causal e sendo responsável por cerca de 99,7\% dos casos. (Souto \& Falhari, [s.d.])

Sobre este patógeno, é de conhecimento que existem mais de 200 tipos descritos na literatura, porém, apenas cerca de 40 deles tem potencial de infecção no trato anogenital. Dentro destes 40, existe uma divisão entre baixo e alto risco. Os de 
baixo risco $(6,11,40,42,43,54,61,70,72,81$, CP6 108) relacionam-se com condilomas e lesões de baixo grau. Já os de alto risco $(16,18,31,33,35,39,45,51,52,56,58,59,68,73$ e 82) associam-se às neoplasias malignas. Para o câncer de colo de útero, especificamente, a maior relação é com os tipos 16 e 18.(FEBRASGO, 2018)

Um estudo realizado com mais de 1000 amostras de pacientes com câncer cervical de 32 hospitais em 22 países identificou o DNA do HPV em 93\% dos tumores, sem variação significativa. O tipo 16 estava presente em $50 \%$ dos espécimes, o 18 em 14\%, o 45 em 8\%, e o 31 em 5\% (Bosch et al., 1995). Corroborando com este achado, uma metanálise acerca da distribuição mundial dos tipos de HPV identificou prevalência de 57\% do HPV 16 e 16\%, do 18 (Li et al., 2011).

Ainda falando sobre os fatores de risco, sabe-se que o início da vida sexual com idade inferior a 16 anos, múltiplos parceiros sexuais e história prévia de condilomas genitais são alguns deles. Pacientes que fazem uso de drogas imunossupressoras, mulheres que usam anticoncepcional oral combinado por tempo prolongado e pacientes tabagistas também se enquadram no grupo de risco para desenvolvimento dessa neoplasia (FEBRASGO, 2017). Além disso, nota-se que mulheres que tiveram filhos antes dos 20 anos de idade e aquelas consideradas multíparas também possuem risco aumentado (The International Collaboration of Epidemiological Studies of Cervical Cancer, 2007). Além disso, pessoas de etnias não brancas aparentam ter uma maior predisposição para o desenvolvimento dessa neoplasia (Saraiya et al., 2007).

O diagnóstico é realizado por meio de colposcopia com biópsia, que são realizadas a depender dos resultados do exame físico e da citologia cervical. A partir daí, exames complementares podem ser pedidos para auxiliar no estadiamento, o qual é realizado pelo protocolo da Federação Internacional de Ginecologia e Obstetrícia (FIGO) (Tsuchiya et al., 2017). O diagnóstico diferencial é feito durante a investigação com outras patologias que causem sangramento anormal, corrimento ou lesão cervical.

A prevenção primária do câncer de colo de útero envolve a educação sexual e o estímulo ao uso de preservativo a fim de evitar a contaminação com o Papiloma Vírus Humano. No entanto, o principal método de prevenção é a vacina contra o HPV, que no Brasil foi instituída em 2014 para meninas e em 2017 para meninos com idade entre 9 a 14 anos, pois a prevenção é mais eficaz quando realizada antes do início da vida sexual. A vacina protege contra até 4 tipos da doença e deve ser tomada em duas doses com intervalo semestral. (INCA, 2018b)

Por falhas no acompanhamento, no diagnóstico, na prevenção ou por características inatas a cada indivíduo, o desenvolvimento de câncer do colo do útero pode ser a via final comum e neste ponto deve-se considerar a escolha terapêutica mais eficaz para cada caso. O tratamento do câncer e das lesões precursoras variam de acordo com a sua classificação, podendo ser adotada conduta expectante ou ablasiva ou de ressecção a depender de cada caso. No caso da conduta ablasiva podem ser escolhidas técnicas de laser e eletrocauterização; quanto à ressecção, considera-se a realização de cirurgia de alta frequência ou a conização a frio.(Tsuchiya et al., 2017)

Apesar dos inúmeros avanços na Oncologia para o diagnóstico e o acompanhamento terapêutico, pouco mudou na visão do câncer como uma das doenças mais ameaçadoras e mortais e atrelada a diversos simbolismos negativos relacionados à morte, sofrimento e solidão. Diante das vivências associadas a possibilidade de finitude da vida, há uma intensa revisão e questionamento de valores pessoais, mudanças de papéis familiares e reavaliação de planos futuros (Gobatto, 2010; Neme \& Lipp, 2010).

Diante desse cenário, sabe-se que o câncer do colo do útero, além dos estigmas do câncer, é uma doença que atinge um órgão repleto de simbolismos para a mulher, pois envolve questões inerentes à sexualidade, feminilidade e reprodução. A mulher, ao receber o diagnóstico de câncer, vivencia a expectativa de um futuro incerto, tratamentos longos e dolorosos, e também o medo da morte e mutilação (Padilha et al., 2017; Panobianco et al., 2012) As pacientes enfrentam expressões emocionais como inadequação pessoal, ansiedade quanto à possibilidade de recidiva e de infertilidade, incertezas sobre o futuro e depressão.(Biglia et al., 2010; Emilee et al., 2010). 
Apesar disso, aspectos associados à sexualidade não são pautados no contexto clínico, que converge para a eficiência e eficácia do tratamento, para efeitos adversos não sexuais e sobrevida do (a) paciente (Mercadante et al., 2010). Entretanto, a sexualidade é uma questão importante, pois, de acordo com a Organização Mundial das Nações Unidas, é um dos pilares para a qualidade de vida dos indivíduos (Oosterhoff \& Sweetman, 2018).

Sabendo disso, a sexualidade não é um conceito que pode ser considerado separado da saúde, pois consiste em um fator central para a manutenção do bem-estar e do autoconceito. Entretanto, a maioria das mulheres não percebem a sexualidade dentro desse contexto amplo. Muitas delas tem uma visão da sexualidade centrada apenas nos órgãos genitais, o que reduz o conceito e o significado delas a apenas o ato da penetração em si (Ferreira et al., 2013; Trindade \& Ferreira, 2008).

Tendo em vista essa situação, sabe-se também que grande parcela da população não possui êxito nesse aspecto da vida. Estima-se que entre 40 e $45 \%$ das mulheres e 20 a $30 \%$ dos homens têm alguma queixa de disfunção sexual. Entre mulheres com queixas, a prevalência de desejo sexual hipoativo varia de 32 a $58 \%$ e a disfunção de excitação e anorgasmia giram em torno de 30\% (Hayes et al., 2008; Tozo \& Aoki, 2007). Essas disfunções sexuais podem ser decorrentes de fatores físicos, psicológicos ou podem estar associadas a um conjunto de fatores. Estudos demonstram que um mal físico, muitas vezes, acarreta problemas psicológicos, como medo, ansiedade ou tensão e, esses, por sua vez, frequentemente agravam um problema pré-existente (Faubion \& Rulo, 2015)

Dessa maneira, um grande desafio é atender às questões relacionadas à sexualidade da paciente e do(a) parceiro(a), para que ambos possam desenvolver aceitação e adaptação às alterações provocadas pelo câncer e aos impactos dos tratamentos (Fleury et al., 2011). Para isso, se faz necessário um amplo conhecimento e domínio do assunto por parte dos profissionais da saúde, em especial os ginecologistas. (Tozo \& Aoki, 2007)

Dentre os pacientes que enfrentam esse problema, os pacientes oncológicos em geral são uma grande parcela. Vários tipos de cânceres e seus respectivos tratamentos frequentemente se associam a alguma disfunção sexual. Tanto em homens quanto em mulheres, a quimioterapia afeta a função gonadal, levando à inibição do desejo e da excitação, anorgasmia, perda da sensação de bem-estar e da reação a estímulos prazerosos. Em mulheres, a falência da função ovariana pode induzir, inclusive, à menopausa prematura (Oncoguia, 2016a; Vassão et al., 2018).

Focando especificamente nas pacientes com câncer do colo do útero, o tratamento provoca alterações na anatomia e na função vaginal. Ocorrem também modificações na pelve que podem levar a alteração do tamanho e/ou do formato dos órgãos genitais, dificuldade de penetração, rebaixamento do impulso sexual e do grau de atratividade, decorrentes de alterações circulatórias (Cavalcanti et al., 2014; Oncoguia, 2016b).

As cirurgias frequentemente causam prejuízo à inervação e ao suprimento vascular dos órgãos pélvicos, o que afeta a resposta fisiológica ao estímulo sexual, tanto em homens quanto em mulheres. A produção de esteróides sexuais é afetada em casos de orquiectomia, ooforectomia e adrenalectomia, diminuindo o interesse por sexo (Hughes, [s.d.]; Vassão et al., 2018)

Nesse casos, a radiação pélvica também causa disfunção sexual significativa, incluindo ondas de calor, secura vaginal, maior dificuldade em atingir a excitação sexual, lubrificação vaginal, e redução da satisfação sexual global (Froeding et al., 2014; Oncoguia, 2016b) Os pacientes tratados com radioterapia relataram forte efeito negativo sobre a atividade sexual e sobre o funcionamento sexual / vaginal que os pacientes tratados sem irradiação (Mirabeau-Beale \& Viswanathan, 2014).

Assim, este trabalho objetiva avaliar o impacto do câncer do colo do útero sobre a sexualidade de mulheres, compreendendo os sentimentos de impotência e fracasso. Almeja-se, ainda, fornecer aos estudantes da área de saúde, médicos e organizações de câncer sugestões específicas sobre as necessidades de informação sexual depois do câncer e as modalidades que são preferidas, para prevenir e minorar a aflição da paciente. 


\section{Metodologia}

Estudo epidemiológico, descritivo, qualitativo, com coleta de dados de caráter transversal. O universo amostral da pesquisa foi de 293 pacientes diagnosticadas com câncer de útero, conforme quantitativo de prontuários (2010 a 2014) disponibilizados no Hospital de Urgências de Sergipe (HUSE). No entanto, optou-se pela amostra de conveniência uma vez que as pacientes que procuram a unidade hospitalar eram de diferentes locais, dificultando assim a localização censitária.

Foram realizadas visitas ao ambulatório de oncologia do Hospital de Urgência de Sergipe (HUSE) para identificação das pacientes. A coleta de dados foi realizada por pesquisadora treinada, através de questionários individuais.

O primeiro questionário aborda idade, escolaridade, profissão, estado civil, número de filhos e local de residência (Apêndice A).

O segundo consiste no Quociente Sexual versão feminina (QS-F), designado a avaliar a atividade sexual da mulher, existindo também o instrumento destinado a avaliar a sexualidade do homem. Compõe-se de 10 questões, cada qual devendo ser respondida numa escala de 0 a 5 (Anexo A) (ABDO, 2009).

$\mathrm{O}$ resultado da soma das 10 respostas é multiplicado por dois, o que resulta num índice total que varia de $0 \mathrm{a} 100$. A sétima questão requer tratamento diferente, ou seja, o valor da resposta dada (de 0 a 5 ) deve ser subtraído de 5 para se ter o escore final dessa questão. Os valores maiores indicam melhor desempenho/satisfação sexual, a saber: 82-100 pontos: bom a excelente; 62-80 pontos: regular a bom; 42-60 pontos: desfavorável a regular; 22-40 pontos: ruim a desfavorável; 0-20 pontos: nulo a ruim (ABDO, 2009).

Os dados coletados foram tabulados no programa estatístico Epi-Info versão 7.0. Posteriormente foram exportados para o SPSS. As participantes do estudo foram caracterizadas por frequências (absoluta e relativa) para os dados categóricos e, de acordo com a normalidade das variáveis contínuas, em médias ou medianas com seus respectivos desvios padrões ou intervalos de confiança.

Por tratar-se de uma pesquisa com seres humanos, a mesma foi submetida à aprovação pelo Comitê de Ética em Pesquisa da Universidade Tiradentes/SE, sob protocolo n 989.186 (Anexo B) e respeitou as normas da Resolução 466/2012 do Conselho Nacional de Saúde (CNS). Ademais, a todas as pacientes que consentiram em participar da pesquisa foram elucidados acerca dos aspectos relevantes e assinaram o Termo de Consentimento Livre e Esclarecido (TCLE).

\section{Resultados e Discussão}

Para fins de contemplação da amostra, foram entrevistadas 80 mulheres no Hospital de Urgências de Sergipe (HUSE) que se enquadravam nos critérios de inclusão e exclusão adotados.

Evidenciou-se média de idade de 46,8 anos ( $\pm 13,2)$, com valores máximo e mínimo respectivamente de 24 e 88 anos (variação de 64 anos). Essa média de idade corrobora com a literatura, uma vez que de acordo com o Instituto Nacional do Câncer a maioria dos casos acontece a partir dos 30 anos com uma prevalência aumentando com o passar dos anos (INCA, 2018c).

Quanto ao número de filhos a média foi de $3,4( \pm 2,4)$, variando de 0 (zero) a 13 filhos. O que também ratifica a literatura existente, uma vez que ter mais filhos se enquadra como fator de risco para desenvolvimento da doença (Saraiya et al., 2007). 
Tabela 1. Caracterização das mulheres integrantes da amostra. Aracaju, 2015.

\begin{tabular}{|c|c|c|c|}
\hline & & $\mathrm{N}$ & $\%$ \\
\hline \multirow[t]{8}{*}{ Escolaridade } & Analfabeto & 19 & 23,8 \\
\hline & Lê e escreve & 2 & 2,5 \\
\hline & Fundamental completo & 6 & 7,5 \\
\hline & Fundamental incompleto & 18 & 22,5 \\
\hline & Médio completo & 20 & 25,0 \\
\hline & Médio incompleto & 11 & 13,8 \\
\hline & Superior completo & 3 & 3,8 \\
\hline & Superior incompleto & 1 & 1,3 \\
\hline \multirow[t]{7}{*}{ Estado Civil } & Casada & 29 & 36,3 \\
\hline & Com companheiro & 5 & 6,3 \\
\hline & Divorciada & 9 & 11,3 \\
\hline & Separação de corpos & 3 & 3,8 \\
\hline & Solteiro & 25 & 31,3 \\
\hline & União estável & 4 & 5,0 \\
\hline & Viúva & 5 & 6,3 \\
\hline \multirow[t]{3}{*}{ Nível Socioeconômico } & Baixo & 25 & 31,3 \\
\hline & Médio/baixo & 14 & 17,5 \\
\hline & Médio & 41 & 51,2 \\
\hline \multirow[t]{7}{*}{ Profissão } & Aposentada & 7 & 8,8 \\
\hline & Autônoma & 7 & 8,8 \\
\hline & Do lar & 27 & 33,8 \\
\hline & Doméstica & 10 & 12,4 \\
\hline & Lavradora & 12 & 15,0 \\
\hline & Serviços gerais & 7 & 8,8 \\
\hline & Outras profissões & 10 & 12,4 \\
\hline
\end{tabular}

Fonte: Autores (2020).

A escolaridade apresentou-se de maneira heterogênea com prevalência de $25 \%$ das pacientes com nível médio completo $(n=20), 23,8 \%$ das pacientes analfabetas $(n=19), 22,5 \%$ das pacientes com ensino fundamental incompleto $(n=18)$, 13,8\% das pacientes com ensino médio incompleto $(\mathrm{n}=11), 6,5 \%$ das pacientes com ensino fundamental completo $(\mathrm{n}=6)$, $3,8 \%$ das pacientes com ensino superior completo $(n=3), 2,5 \%$ das pacientes apenas alfabetizadas $(n=2)$ e $1,3 \%$ das pacientes com nível superior incompleto $(\mathrm{n}=1)$.

Essa situação nos mostra que 56,25\% das pacientes do grupo amostral largaram a escola antes ou chegaram no máximo até o ensino fundamental ( $\mathrm{n}=45)$, o que as enquadra como baixa escolaridade. Dessa maneira, os dados obtidos nesse estudo concordam com a literatura, uma vez que estudos mostraram que a baixa escolaridade ( 0 a 7 anos de estudo) era maioria entre as pacientes observadas (Thuler et al., 2014).

Quanto ao estado civil, 36,3\% declaram-se casadas ( $\mathrm{n}=29), 31,3 \%$ das pacientes se declaram solteiras $(\mathrm{n}=25), 11,3 \%$ das pacientes são divorciadas $(n=9), 6,3 \%$ dizem que tem um companheiro $(n=5)$, outras 6,3\% das pacientes são viúvas $(n=5)$, $5 \%$ das pacientes dizem que estão em união estável $(\mathrm{n}=4)$, e os outros 3,8\% dizem que estão em separação de corpos ( $\mathrm{n}=3$ ). 
Essa situação também enfatiza a bibliografia já existente, uma vez que estudos mostram maior prevalência de câncer de colo de útero entre mulheres casadas (Mascarello et al., [s.d.]; Thuler et al., 2014)

No que diz respeito ao nível sócio econômico 31,3\% das pacientes se declaram de baixa renda $(\mathrm{n}=25), 17,5 \%$ das pacientes dizem ser de classe média-baixa $(n=14)$ e 51,2\% afirmam ser de classe média $(n=41)$. Essa realidade nos mostra que, do grupo todo, nenhuma paciente se enquadra em classe média-alta ou alta, o que corrobora com a literatura existente. Isso porque, um estudo de Fonseca et al. Evidenciou que a renda média pessoal das pacientes avaliadas era de 1,1 salário mínimo, ou seja, classe média ou média-baixa (Fonseca et al., 2010). Além disso, de acordo com a Organização Mundial da Saúde, mais de $85 \%$ dos casos estão localizados em países de menor renda.

À respeito da profissão, 33,8\% afirmam ser do lar $(\mathrm{n}=27), 15 \%$ responderam que são lavradoras $(\mathrm{n}=12), 12,4 \%$ se declararam domésticas $(n=10), 12,4 \%$ dizem ser de outras profissões $(n=10), 8,8 \%$ afirmam ser aposentadas ( $n=7)$, outras $8,8 \%$ são autônomas $(n=7)$ e as demais $8,8 \%$ trabalham com serviços gerais $(n=7)$. Essa realidade concorda com a literatura, uma vez que um estudo realizado por Fonseca et al. mostrou como atividade profissional mais comum o emprego doméstico (“do lar”) (Fonseca et al., 2010). e renda pessoal média de 1,1 salário mínimo, sendo portanto classificadas como classe média ou média baixa.

Com relação ao QS-F foi evidenciado média geral de 45,5 pontos $( \pm 23,9)$, variando de 10 (zero) a 84 pontos. Sabendo que a classificação dos resultados tem 5 variações (nulo-ruim, ruim-desfavorável, desfavorável-regular, regular-bom, bom excelente) e que essas variações correspondem, respectivamente, aos intervalos 0-20, 22-40, 42-60, 62-80 e 82-100 pontos, nota-se que a média das pacientes analisadas se enquadra na categoria “desfavorável-regular”.

Essa realidade mostra que uma parcela dos indivíduos pesquisados não estão tendo êxito nesse aspecto e podem ter sua qualidade de vida prejudicada, como mostra também a bibliografia (Hayes et al., 2008). Além disso, nos permite inferir que um mal físico, como o câncer de colo de útero, pode alterar o psicológico, emocional e autoaceitação do corpo, comprometendo também a sexualidade das pacientes (Faubion \& Rulo, 2015).

Comparando as proporções das classificações das pontuações obtidas por meio do QSF de acordo com a situação conjugal das entrevistadas pode-se vislumbrar o apresentado na Tabela 2 abaixo:

Tabela 2. Distribuição das proporções da classificação das pontuações do QSF de acordo com a situação conjugal, Aracaju, 2015.

\begin{tabular}{|c|c|c|c|c|c|c|c|c|c|c|}
\hline & \multicolumn{3}{|c|}{ Sem Companheiro } & \multicolumn{3}{|c|}{ Com companheiro } & \multicolumn{3}{|c|}{ Ambos } & \multirow[t]{2}{*}{$\mathrm{p}$} \\
\hline & $\mathrm{n}$ & $\%$ & $\%^{\mathrm{a}}$ & $\mathrm{n}$ & $\%$ & $\%^{\mathrm{a}}$ & $\mathrm{N}$ & $\%$ & $\%^{\mathrm{a}}$ & \\
\hline Nulo/ruim & 12 & 15,0 & 15,0 & 10 & 12,5 & 12,5 & 22 & 27,4 & 27,4 & \\
\hline Ruim/desfavorável & 4 & 5,0 & 20,0 & 4 & 5,0 & 17,5 & 8 & 10,0 & 37,4 & \\
\hline Desfavorável/regular & 16 & 20,0 & 40,0 & 15 & 18,7 & 36,2 & 31 & 38,8 & 76,2 & 0,22 \\
\hline Regular/bom & 8 & 10,0 & 50,0 & 8 & 10,0 & 46,2 & 16 & 20,0 & 90,2 & \\
\hline Bom/excelente & 2 & 2,5 & 52,5 & 1 & 1,3 & 47,5 & 3 & 3,8 & 100,0 & \\
\hline
\end{tabular}

$\%^{\mathrm{a}}$ - Frequência relativa acumulada. Fonte: Autores (2020).

Adotada a classificação proposta pelos idealizadores do instrumento e observada a frequência relativa acumulada, nota-se que 76,2\% (n=61) foram classificadas abaixo da categoria "desfavorável/regular". Desta totalidade dos classificados na supracitada categoria, $40 \%(\mathrm{n}=32)$ não possuíam companheiros, todavia, a melhor pontuação também foi obtida por pacientes sem companheiro $2,5 \%(\mathrm{n}=2)$. 
Seguindo a estratificação preconizada, levando-se em consideração o nível socioeconômico, a Tabela 3 abaixo apresenta os resultados:

Tabela 3. Distribuição das proporções da classificação das pontuações do QSF de acordo com o nível socioeconômico, Aracaju, 2015.

\begin{tabular}{|c|c|c|c|c|c|c|c|c|c|c|}
\hline & \multicolumn{3}{|c|}{ Baixo } & \multicolumn{3}{|c|}{ Médio/baixo } & \multicolumn{3}{|c|}{ Médio } & \multirow[t]{2}{*}{$\mathrm{p}$} \\
\hline & $\mathrm{n}$ & $\%$ & $\%^{a}$ & $\mathrm{n}$ & $\%$ & $\%^{\mathrm{a}}$ & $\mathrm{N}$ & $\%$ & $\%^{\mathrm{a}}$ & \\
\hline nulo/ruim & 10 & 12,5 & 12,5 & 5 & 6,1 & 6,1 & 7 & 8,8 & 8,8 & \multirow{6}{*}{$<0,05^{*}$} \\
\hline ruim/ desfavorável & 3 & 3,8 & 16,3 & 4 & 5,0 & 11,1 & 1 & 1,3 & 10,1 & \\
\hline desfavorável/ regular & 7 & 8,8 & 25,1 & 4 & 5,0 & 16,1 & 20 & 25,0 & 35,1 & \\
\hline regular/bom & 2 & 2,5 & 27,6 & 1 & 1,2 & 17,3 & 13 & 16,2 & 51,3 & \\
\hline bom/ excelente & 3 & 3,8 & 31,4 & 0 & 0 & 0 & 0 & 0 & 0 & \\
\hline Total & 25 & 31,4 & & 14 & 17,3 & & 41 & 51,3 & & \\
\hline
\end{tabular}

\% - Frequência relativa acumulada; *Associação estatisticamente significativa a nível de 0,001, exato de Fisher. Fonte: Autores (2020).

A baixa pontuação advinda do QS-F pode estar associada às respostas afetivas ligadas às condições impostas pela doença. Um estudo realizado por Bomfim et al. com mulheres mastectomizadas, evidenciou desempenho sexual de nulo a ruim em 35,3\% ( $n=12)$ das mulheres, e apenas $11,8 \%(n=4)$ com escore favorável. Essa pesquisa mostrou que $27,4 \%$ foram classificadas com o pior escore e apenas 9,8\% com o melhor (Quintiliano Montenegro Bomfim et al., 2014).

\section{Conclusão}

Os resultados encontrados nesse estudo mostram que, de acordo com a epidemiologia e condições sociodemográficas, o grupo amostral é semelhante em vários aspectos com os estudos comparados.

Além disso, de acordo com os resultados encontrados no estudo, observa-se que a maioria das mulheres acometidas por câncer do colo do útero investigadas mostra função sexual baixa e insatisfatória de acordo com o QS-F. Vale ressaltar que essa disfunção sexual é resultado de uma série de fatores que também foram bem detalhadas no trabalho.

Os resultados evidenciados por esta pesquisa são preocupantes e devem ser alvo de medidas corretivas em tempo hábil. A depreciação da imagem corporal, baixa autoestima e desconfiança são fatores que podem ser minimizados por medidas simples como a realização de grupos de discussão e diálogo paciente-profissional.

Assim, é necessário que os profissionais de saúde pensem a despeito de como melhor prestar assistência a esta população no intuito de oferecer informações que minimizem os fatores que interferem na função sexual e os estimulem a promover mudanças no estilo de vida para a melhoria da sua qualidade de vida. É essencial que tenham em mente que o amplo espectro de consequências do câncer requer uma abordagem terapêutica também psicológica e holística que contemple a totalidade dos fatores depressores da saúde destas mulheres.

Para além disso, faz-se necessário que mais trabalhos sejam realizados nessa perspectiva tanto de caráter nacional quanto de caráter internacional. Isso se justifica por tamanha importância do assunto e tão pouca literatura atualizada a respeito.

\section{Referências}

Biglia, N., Moggio, G., Peano, E., Sgandurra, P., Ponzone, R., Nappi, R. E., \& Sismondi, P. (2010). Effects of Surgical and Adjuvant Therapies for Breast Cancer on Sexuality, Cognitive Functions, and Body Weight. The Journal of Sexual Medicine, 7(5), 1891-1900. https://doi.org/10.1111/j.17436109.2010.01725.x 
Bosch, F. X., Manos, M. M., Munoz, N., Sherman, M., Jansen, A. M., Peto, J., Schiffman, M. H., Moreno, V., Kurman, R., Shan, K. V., \& International Biological Study on Cervical Cancer (IBSCC) Study Group. (1995). Prevalence of Human Papillomavirus in Cervical Cancer: A Worldwide Perspective. JNCI Journal of the National Cancer Institute, 87(11), 796-802. https://doi.org/10.1093/jnci/87.11.796

Cavalcanti, I. F., Farias, P. da N., Ithamar, L., Silva, V. M. da, \& Lemos, A. (2014). Função sexual e fatores associados à disfunção sexual em mulheres no climatério. Revista Brasileira de Ginecologia e Obstetrícia, 36(11), 497-502. https://doi.org/10.1590/S0100-720320140004985

De Mendonça, F. C., Costa, G. O., \& Ribeiro, A. A. (2019). Prevalência do diagnóstico citopatológico: Uma abordagem sobre as condutas preconizadas pelo Ministério da Saúde. Revista EVS - Revista de Ciências Ambientais e Saúde, 46(1), 17. https://doi.org/10.18224/evs.v46i1.6453

Emilee, G., Ussher, J. M., \& Perz, J. (2010). Sexuality after breast cancer: A review. Maturitas, 66(4), 397-407. https://doi.org/10.1016/j.maturitas.2010.03.027

Faubion, S., \& Rulo, J. (2015). Disfunção Sexual Na Mulher: Uma Abordagem Prática. 3.

FEBRASGO. (2017). Rastreio, Diagnóstico e Tratamento do Câncer de Colo de Útero. (2a ed,), 1. Federação Brasileira de Ginecologia e Obstetrícia. https://www.febrasgo.org.br/media/k2/attachments/05Z-ZDIAGNOySTICOZRASTREIOZEZTRATAMENTOZDOZCAyNCERZDEZCOLOZDEZ UyTERO.pd

FEBRASGO. (2018). Número de Infecções Sexualmente Transmissíveis (IST) aumenta. https://www.febrasgo.org.br/pt/noticias/item/565-numero-deinfeccoes-sexualmente-transmissiveis-ist-aumenta

Ferreira, S. M. de A., Panobianco, M. S., Gozzo, T. de O., \& Almeida, A. M. de. (2013). A sexualidade da mulher com câncer de mama: Análise da produção científica de enfermagem. Texto \& Contexto - Enfermagem, 22(3), 835-842. https://doi.org/10.1590/S0104-07072013000300033

Fleury, H. J., Pantaroto, H. S. de C., \& Abdo, C. H. N. (2011). Sexualidade em oncologia.

Fonseca, A. J. da, Ferreira, L. P., Dalla-Benetta, A. C., Roldan, C. N., \& Ferreira, M. L. S. (2010). Epidemiologia e impacto econômico do câncer de colo de útero no Estado de Roraima: A perspectiva do SUS. Revista Brasileira de Ginecologia e Obstetrícia, 32(8), 386-392. https://doi.org/10.1590/S010072032010000800005

Froeding, L. P., Ottosen, C., Rung-Hansen, H., Svane, D., Mosgaard, B. J., \& Jensen, P. T. (2014). Sexual Functioning and Vaginal Changes after Radical Vaginal Trachelectomy in Early Stage Cervical Cancer Patients: A Longitudinal Study. The Journal of Sexual Medicine, 11(2), 595-604. https://doi.org/10.1111/jsm.12399

Gobatto, C. A. (2010). Coping religioso-espiritual: Reflexões e perspectivas para a atuação do psicólogo em oncologia. 12.

Hayes, R. D., Dennerstein, L., Bennett, C. M., \& Fairley, C. K. (2008). What is the "True" Prevalence of Female Sexual Dysfunctions and Does the Way We Assess These Conditions Have an Impact? The Journal of Sexual Medicine, 5(4), 777-787. https://doi.org/10.1111/j.1743-6109.2007.00768.x

Hughes, M. K. ([s.d.]). ALTERATIONS OF SEXUAL FUNCTION IN WOMEN WITH CANCER. 11.

INCA. (2018a, maio 3). Conceito e Magnitude. INCA - Instituto Nacional de Câncer. https://www.inca.gov.br/controle-do-cancer-do-colo-do-utero/conceitoe-magnitude

INCA. (2018b, maio 3). Prevenção do câncer do colo do útero. INCA - Instituto Nacional de Câncer. https://www.inca.gov.br/controle-do-cancer-do-colodo-utero/acoes-de-controle/prevencao

INCA. (2018c, agosto 22). Estatísticas de câncer. INCA - Instituto Nacional de Câncer. https://www.inca.gov.br/numeros-de-cancer

Li, N., Franceschi, S., Howell-Jones, R., Snijders, P. J. F., \& Clifford, G. M. (2011). Human papillomavirus type distribution in 30,848 invasive cervical cancers worldwide: Variation by geographical region, histological type and year of publication. International Journal of Cancer, 128(4), 927-935. https://doi.org/10.1002/ijc.25396

Mascarello, K. C., Silva, N. F., Piske, M. T., Viana, K. C. G., Zandonade, E., \& Amorim, M. H. C. ([s.d.]). Socio-demographic and Clinical Profile of Women with Cervical Cancer Associated with Initial Staging. 10.

Mercadante, S., Vitrano, V., \& Catania, V. (2010). Sexual issues in early and late stage cancer: A review. Supportive Care in Cancer, 18(6), 659-665. https://doi.org/10.1007/s00520-010-0814-0

Mirabeau-Beale, K. L., \& Viswanathan, A. N. (2014). Quality of life (QOL) in women treated for gynecologic malignancies with radiation therapy: A literature review of patient - reported outcomes. Gynecologic Oncology, 134(2), 403-409. https://doi.org/10.1016/j.ygyno.2014.05.008

Neme, C. M. B., \& Lipp, M. E. N. (2010). Estresse psicológico e enfrentamento em mulheres com e sem câncer. Psicologia: Teoria e Pesquisa, 26(3), 475483. https://doi.org/10.1590/S0102-37722010000300010

Oncoguia, I. (2016a). Mantendo sua Vida Sexual apesar do Tratamento do Câncer. Instituto Oncoguia. http://www.oncoguia.org.br/conteudo/mantendo-suavida-sexual--apesar-do-tratamento-do-cancer/9034/1035/

Oncoguia, I. (2016b). Sexualidade x Câncer de Colo do Útero. Instituto Oncoguia. http://www.oncoguia.org.br/conteudo/sexualidade-X-cancer-de-colo-doutero/8985/566/

Oosterhoff, P., \& Sweetman, C. (2018). Introduction: Sexualities. Gender \& Development, 26(1), 1-14. https://doi.org/10.1080/13552074.2018.1446725 
Research, Society and Development, v. 10, n. 2, e41710212375, 2021

(CC BY 4.0) | ISSN 2525-3409 | DOI: http://dx.doi.org/10.33448/rsd-v10i2.12375

Padilha, C. M. L., Araújo Junior, M. L. C., \& Souza, S. A. L. de. (2017). Cytopathologic evaluation of patients submitted to radiotherapy for uterine cervix cancer. Revista Da Associação Médica Brasileira, 63(4), 379-385. https://doi.org/10.1590/1806-9282.63.04.379

Panobianco, M. S., Pimentel, A. V., de Almeida, A. M., \& Oliveira, I. S. B. (2012). Women Diagnosed with Advanced Cancer of the Cervix: Coping with the Disease and Treatment. 7.

Quintiliano Montenegro Bomfim, I., Santos Batista, R. P., \& Melo de Carvalho Lima, R. (2014). Avaliação da função sexual em um grupo de mastectomizadas. Revista Brasileira em promoção da Saúde, 27(1), 77-84. https://doi.org/10.5020/18061230.2014.p77

Saraiya, M., Ahmed, F., Krishnan, S., Richards, T. B., Unger, E. R., \& Lawson, H. W. (2007). Cervical Cancer Incidence in a Prevaccine Era in the United States, 1998-2002: Obstetrics \& Gynecology, 109(2, Part 1), 360-370. https://doi.org/10.1097/01.AOG.0000254165.92653.e8

Saslow, D., Solomon, D., Lawson, H. W., Killackey, M., Kulasingam, S. L., Cain, J., Garcia, F. A. R., Moriarty, A. T., Waxman, A. G., Wilbur, D. C., Wentzensen, N., Downs, L. S., Spitzer, M., Moscicki, A.-B., Franco, E. L., Stoler, M. H., Schiffman, M., Castle, P. E., Myers, E. R., \& ACS-ASCCP-ASCP Cervical Cancer Guideline Committee. (2012). American Cancer Society, American Society for Colposcopy and Cervical Pathology, and American Society for Clinical Pathology screening guidelines for the prevention and early detection of cervical cancer. CA: A Cancer Journal for Clinicians, 62(3), 147-172. https://doi.org/10.3322/caac.21139

Souto, R., \& Falhari, J. P. B. ([s.d.]). O Papilomavírus Humano: Um fator relacionado com a formação de neoplasias. 6.

The International Collaboration of Epidemiological Studies of Cervical Cancer. (2007). Comparison of risk factors for invasive squamous cell carcinoma and adenocarcinoma of the cervix: Collaborative reanalysis of individual data on 8,097 women with squamous cell carcinoma and 1,374 women with adenocarcinoma from 12 epidemiological studies: Squamous Cell Carcinoma and Adenocarcinoma of the Cervix. International Journal of Cancer, 120(4), 885-891. https://doi.org/10.1002/ijc.22357

Thuler, L. C. S., Aguiar, S. S. de, \& Bergmann, A. (2014). Determinantes do diagnóstico em estadio avançado do câncer do colo do útero no Brasil. Revista Brasileira de Ginecologia e Obstetrícia, 36(6), 237-243. https://doi.org/10.1590/S0100-720320140005010

Tozo, I. M., \& Aoki, T. (2007). Disfunção sexual feminina: A importância do conhecimento e do diagnóstico pelo ginecologista. 6.

Trindade, W. R., \& Ferreira, M. de A. (2008). Sexualidade feminina: Questões do cotidiano das mulheres. Texto \& Contexto - Enfermagem, 17(3), 417-426. https://doi.org/10.1590/S0104-07072008000300002

Tsuchiya, C., Lawrence, T., Klen, M., Fernandes, R., \& Alves, M. R. (2017). O câncer de colo do útero no Brasil: Uma retrospectiva sobre as políticas públicas voltadas à saúde da mulher. Jornal Brasileiro de Economia da Saúde, 9(1), 137-147. https://doi.org/10.21115/JBES.v9.n1.p137-47

Vassão, F. V., Barbosa, L. R., Moraes, G. M. de, \& Domenico, E. B. L. D. (2018). Abordagem da sexualidade no cuidado ao pacie nte oncológico: Barreiras e estratégias. Acta Paulista de Enfermagem, 31(5), 564-571. https://doi.org/10.1590/1982-0194201800078

World Health Organization. (2017, fevereiro 6). OMS: 85\% das mortes por câncer de colo de útero ocorrem em países de média e baixa renda. ONU Brasil. https://nacoesunidas.org/oms-85-das-mortes-por-cancer-de-colo-de-utero-ocorrem-em-paises-de-media-e-baixa-renda/ 